\title{
Use of Anti-Inflammatory Drugs interventions for the treatment of Muscle Soreness: a Systematic Review and Meta-analysis.
}

Roberto Lohn Nahon

Laboratory of Protein Biochemistry - Federal University of State of Rio de Janeiro (UNIRIO), RJ, Brazil. https://orcid.org/0000-0002-0186-9846

Anibal Monteiro de Magalhães Neto

Federal University of Mato Grosso (UFMT), Barra do Garças, MT, Brazil

Jaqueline Santos Silva Lopes

Federal University of Mato Grosso (UFMT), Barra do Garças, MT, Brazil.

Aloa de Souza Machado

Federal University of Rio de Janeiro (UFRJ), Laboratory of Experimental Toxicology (LaTEx), Department of Clinical and Toxicological Analysis, Faculty of Pharmacy, Rio de Janeiro, RJ, Brazil.

L. C. Cameron ( $\square$ cameron@unirio.br)

Laboratory of Protein Biochemistry - Federal University of State of Rio de Janeiro (UNIRIO), RJ, Brazil. https://orcid.org/0000-0003-4180-2061

\section{Systematic Review}

Keywords: Pharmacology, Pain, Sports Medicine, Evidence-Based Medicine, non-steroidal anti-inflammatory drug, Delayed Onset Muscle Soreness (DOMS)

Posted Date: August 31st, 2020

DOI: https://doi.org/10.21203/rs.3.rs-67287/v1

License: @ (i) This work is licensed under a Creative Commons Attribution 4.0 International License. Read Full License

Version of Record: A version of this preprint was published at Revista Brasileira de Medicina do Esporte on August 31st, 2020. See the published version at https://doi.org/10.1590/1517-8692202127062021_0072. 


\section{Abstract}

Objective: To investigate the effects of pharmacological interventions in the treatment of Delayed Onset Muscle Soreness (DOMS).

Design: Systematic review and meta-analysis of randomised controlled clinical trials (RCTs).

Data sources: The PubMed / MEDLINE, EMBASE, SPORTDiscus, Scielo and CENTRAL (Cochrane Central Register of Controlled Trials) databases were searched from the oldest records to August 3, 2020.

Eligibility criteria: 1) Tue used a RCTs design; 2) Evaluate the effects of Steroidal or Non-Steroidal Anti-Inflammatory Drugs (NSAIDs) for treatment DOMS; and 3) Therapeutically used drugs, after exercise.

Results: In total, 26 studies (patients $=934$ ) were eligible for qualitative analysis on the treatment of DOMS. The results of the meta-analysis showed no superiority between the use or not of NSAIDs, in the improvement of late muscle pain, since statistically significant differences were not verified (21 studies, $\mathrm{n}=$ $955 ; \mathrm{SMD}=0.02 ; 95 \% \mathrm{Cl}-0.58,0.63 ; \mathrm{p}=0.94 ; \mathrm{I}=93 \%$ ). The quality of the synthesized evidence was very low according to the criteria of Evaluation, Development and Evaluation of the Classification of Recommendations, associated with the significant heterogeneity among the included studies.

Conclusion: The results demonstrate that the use of NSAIDs is not a superior treatment to the control / placebo on DOMS improvement. The variation between dose-response and exercise protocol used in the studies may have influenced the results. In addition, the high risk of identified bias characterizes limitation to be considered in profound interpretations.

\section{Introduction}

The excess of exercise for a given physical conditioning can cause inflammation. Strenuous and unusual exercises can cause sub-macroscopic tissue damage, which is associated with symptoms such as stiffness, impairment of range of motion and discomfort. These events normally result in a late-onset muscle pain, known as Delayed Onset Muscle Soreness (DOMS) and are responsible for impairing sports performance [1]. Pain is not perceived either during or right after exercise, but generally happens in a $24-48$ hours period [2, 3]. The inflammatory response developed after exercise characterizes a process for tissue recovery and is related to muscle recovery and adaptation essential for the functional gain [3]. Pain constitutes an unpleasant experience, which limits daily activities. And its treatment is the aim of both the prescriber and the patient. Thus, the use of non-steroidal anti-inflammatory drugs (NSAIDs) are commonly suggested to contain pain and improve the recovery process.

NSAIDs act by inhibiting cyclooxygenase family (EC 1.14.99.1) enzymes. Leading to the decrease of prostaglandins, prostacyclins and thromboxane synthesis. The decrease of prostaglandins concentration reduces acute inflammation, lowering pain neural pathways and inhibiting installation of edema [4]. It is well known that NSAIDs blocks mTOR signaling [5]. Consequently, the use of NSAIDs may suppress myofibril regeneration as well as cell proliferation or differentiation and hypertrophy $[4,6]$.

Previous studies have shown ambiguous data on the use of NSAIDs in DOMS. Ibuprofen decreases macrophage infiltration in the damaged tissue within 24 hours after exercise [7]. On the other hand, the use of naproxen did not alter tissue infiltration of inflammatory cells after experimental muscle damage protocol [8].

Vella et al. (2016) propose that NSAIDs decreases the intensity of the inflammatory response and leukocyte infiltration in skeletal muscle. Their hypothesis reinforces that the intensity of exercise and tissue responses influence the clinical and side effects of anti-inflammatory drugs used to treat DOMS [9].

About the pain, one classical sing of inflammation, clinical trials using NSAIDs showed effect decreasing pain related to exercise when the use of diclofenac [10] and, also, with ibuprofen [11].

There are conflicting data about the use of NSAIDs for the treatment of DOMS. Some reports show decrease of pain and others report the impairment in the process of adaptation or function and the lack of effect in pain $[12,13]$. Thus, more studies need to be done to enlighten this apparent contradiction. The consideration of the dose-response, population profile and type of exercise must be associated with therapy. And more the use of personalized medicine can be a way to help the understanding of the different responses to NSAIDs in different exercise protocols [14]

The clinical management of DOMS involves the attenuation of the inflammatory process, reducing both function and performance. Despite the various NSAIDs options used for the treatment of DOMS, little is known about the magnitude of their clinical effects, mostly due to the use of different protocols. An 
additional concern is the high frequency of adverse reactions resulting from the use of these drugs. These collateral effects are worsened by the indiscriminate use without a medical recommendation [15].

Due to the many pharmacological options and the complexity to the management of DOMS, a review may be useful to assist in understanding the clinical control of DOMS. Therefore, the objective of the present review and meta-analysis study was to investigate the effects of NSAID-type pharmacological interventions in the treatment of DOMS.

\section{Methods}

The Preferred Reporting Items for Systematic Reviews and Meta-Analyses (PRISMA) was used as a guideline [16, 17]. This review was registered in the International Prospective Register of Systematic Reviews (PROSPERO). We analyzed a total of 13,497 studies retrieved from different databases and one from the references on that studies [18].

\section{Study search and selection strategy}

We performed a broad search of keywords and terms related to DOMS, late muscle pain and anti-inflammatory drugs were combined to search in major databases. We used PubMed/MEDLINE, EMBASE, SPORTDiscus, Scielo and CENTRAL (Cochrane Central Register of Controlled Trials) to review all the manuscripts until August 03, 2020. In addition, a manual search in the references of all included studies was performed in order to add the electronic searches. A summarized description of this process is showed in Figure 1.

\section{Inclusion and exclusion criteria}

The next processes took place in stages (title, abstract and full text). We included studies that: 1) used a randomized controlled clinical trials (RCTs) design; 2) evaluated the effects of NSAIDs for treatment DOMS; and 3) analyzed therapeutic drugs after exercise. Case reports, case series, comments, editorials, letters to the editor and literature reviews were excluded. There were no restrictions regarding: age, gender, clinical condition, level of activity, date of publication or language. Both pathological and healthy clinical conditions were considered for selection. We only included studies with healthy participants, free of acute or chronic diseases. Both the detailed search strategy used can be found in supplemental material. For the purpose of this review and metaanalysis we did not seek studies related to steroidal anti-inflammatory drugs.

Figure 1. Description of excluded studies according to the established criteria.

\section{Data extraction}

We collected the following information after selecting the eligible studies: (1) general characterization of the study (authors; year of publication and design), (2) data of the studied population (sample size; gender distribution and age), (3) information related to late muscle pain (the protocol used for inducing muscle damage; type of intervention; dose-response; the method for assessing pain intensity; evaluations timeframe) and (4) outcomes of clinical pain improvement. The corresponding author of the studies was contacted to provide clarification in the case of lack of information.

\section{Risk of bias assessment}

The risk of bias was investigated for each analyzed study. The following items were considered and reported: potential selection bias (regarding sequence and concealment of allocation), performance bias (blinding of subjects and researches), detection bias (blinding evaluation of results), friction bias (incomplete result data), report bias (selective result report), and other bias. Thus, for each item described, the studies received possible ratings: low, high or unclear risk (when the information presented in the study was not sufficient to assess a particular area) [19].

Inclusion and exclusion criteria; data extraction and Risk of bias assessment were simultaneously analyzed by two independent authors using the Cochrane Collaboration Risk of Bias Tool [17, 20]. The data were analyzed using Review Manager (RevMan, 5.3.5). 


\section{Statistical analysis}

The data were grouped in meta-analysis and reported as standardized mean difference (SMD) with 95\% confidence interval (CI). The random-effect model was adopted due to the heterogenicity of the studies $\left(I^{2}=93 \%\right)$ and reported as the value of $\mathrm{I}^{2}$. We included 19 studies for meta-analysis. Seven studies were excluded because of the use of visual analogue scale and three that presented incomplete data (the authors did not provide the requested information).

\section{Results}

Due to the different denominations of DOMS in this review we treated late-onset muscle pain and Delayed Onset Muscle Soreness (DOMS) as synonyms for both analyzes and discussion. A broad selection of papers retrieved 13,497 studies. A total of 127 investigations were considered eligible after applying the criteria (Fig 1). Of these, 23 studies were excluded for not using NSAIDs-type pharmacological interventions; 36 were excluded for not using the intervention after the effort and 42 studies were excluded because of the use of supplements, hormones or homoeopathy. We did not seek studies related to steroidal antiinflammatory drugs. At the end we included 26 studies that met the proposed criteria (Table 1).

We analyzed characteristics of the subjects and studies and summarized them in Table 1. We retrieved three decades of studies starting in 1988 . The majority of the studies were performed in parallel groups protocol (65.4\%), some with cross-over (30.8\%) and a minority of counter-balanced (3.8\%). A total of 934 subjects were studied (18-70 years, mean and SD $=35.9 \pm 34.2 \mathrm{yrs}$ ), from these $55.0 \%$ male. The subjects were described as trained $(15.4 \%)$ or physically active - healthy $(84.6 \%)$.

The majority of the studies were carried out in North America (57.7\%): United States [7, 10, 21-30]; Canada [8, 31, 32]; Europe (34.6\%): United Kingdom [33-35]; Germany [36, 37]; Greece [11]; Denmark [18, 38]; Belgium [39];Africa (3.8\%): South Africa [40] and Oceania (3.8\%): Australia [9].

Concerning sample size, 13 articles (51.8\%) included surveyed samples up to 20 participants, 12 studies (44.4\%) had between 21 and 100 participants, and one study included more than 100 participants (3.8\%). The majority of the studies (57.7\%) only men, while other studies included both sexes.

The protocols used in the studies for inducing muscle damage varied both on the anatomical region and the type of equipment used for evaluation. Thus, in relation to the anatomical site, the studies varied between systemic protocols $(23.1 \%)[24,29,33,34,37,40]$ or localized, in the latter case 8 studies (30.8\%) applied upper limb damage protocol [18, 21, 22, 25, 28, 30, 32, 35]; 11 studies (42.2\%) lower limbs [7-11, 23, 26, 27, 31, 37] and one study (3.8\%) with exercise in the temporomandibular joint [33]. Regarding the equipment used for comparation of the results, two studies (7.7\%) used the isokinetic dynamometer [37, 39], 17 studies $(65,4 \%)$ used conventional weight machines [7-11, 18, 21-23, 25-28, 31, 32, 35] and 6 studies (23.1\%) performed aerobic exercises, lasting more than 30 minutes $[24,29,33,34,37,40]$.

NSAIDs are classified according to their selectivity to cyclooxygenase (COX) 2 inhibition. We found that 23 studies that used non-selective inhibitors (88.4\%), while two studies investigated selective models, (7.6\%). One study [24] did not concern about the type of NSAIDs used, since that the participants were free to use their choice of NSAIDs.

It was observed that the studies varied in the types of non-selective NSAIDs used, with more than half of the studies investigating ibuprofen (56.0\%) [7, 9, 11, $18,22,23,25,28-30,32,34,35,38]$. Other types used were naproxen (12.0\%) [8, 26, 31]; diclofenac (8.0\%) [10, 33]' ketoprofen (8.0\%)[27, 36]; acetaminophen $(8 \%)[7,21]$; aspirin $(4.0 \%)[21]$ and piroxican (4\%) [39].

The major route of administration was oral $(77.0 \%)[6,7,8,10,17,20,21,22,23,25,27,29,28,30-34,36,37,38,39]$. Some studies analyzed topical $(11.5 \%)[9,24,26]$ ' or both $(11.5 \%)[23,35,37]$. Treatment beginning after the effort and remaining for different periods of time, with a maximum duration of seven days.

Thirteen studies (50.0\%) did not find significant effects on the oral use of non-selective NSAIDs for the treatment of DOMS, while ten (38.5\%) considered positive outcomes. All studies that used topical route had good outcomes on DOMS. 
Regarding the two studies investigating selective NSAIDs, one used etoricoxib [37] (90mg/day for 7 days) and the other, rofecoxib [40] (50mg/day for 3 days). In both studies no significant effect was found.

The evaluation of pain was assessed by either visual analogue scale (82.2\%) and mechanical pain (17.8\%). Different moments of pain were evaluated in the studies. Most commonly, the follow-up started before the effort (baseline). Also, different follow-ups were used ranging from 24 hours to 7 days.

\section{Risk of bias assessment}

The bias risk assessment for each study is presented in Figure 2. As observed, the studies were prone to expose the following percentages of low risk of bias random sequence generation ( $80.9 \%)$, allocation concealment (4.7\%), blinding of participants and personnel (71.4\%), blinding of outcome assessment (14.2\%), incomplete outcome data (33.3\%), selective reporting (0\%) and other bias $(42.8 \%)$.

Figure 2. Bias risk evaluation of the selected studies examining the efficacy of NSAIDs for muscle soreness. Low risk (+), unclear risk (blank) and high risk (-) for different features of the Cochrane Risk of Bias Tool. 


\begin{tabular}{|c|c|c|c|c|c|c|c|c|c|}
\hline Year & Design & Subjects & $\begin{array}{l}\text { Exercise } \\
\text { Protocol* }\end{array}$ & $\begin{array}{l}\text { Drugs and } \\
\text { route of } \\
\text { administration }\end{array}$ & Dose & & Assessement & Assessement protocol & $\begin{array}{l}\text { Results } \\
\text { Conclus }\end{array}$ \\
\hline $\begin{array}{l}\text { Arendt et } \\
\text { al., } 2007\end{array}$ & $\begin{array}{l}\text { Parallel } \\
\text { groups }\end{array}$ & $\begin{array}{l}\mathrm{n}=60 \\
60 \text { men } \\
\text { training level: } \\
\text { "healthy right- } \\
\text { handed } \\
\text { Caucasian } \\
\text { subjects" } \\
24.3 \pm 3.1 \text { yrs }\end{array}$ & $\begin{array}{l}\text { Intensive } \\
\text { eccentric } \\
\text { exercise of the } \\
\text { first dorsal } \\
\text { interosseous } \\
\text { muscle of the } \\
\text { left hand on a } \\
\text { standardized } \\
\text { hand exerciser } \\
\text { for two } \\
\text { minutes }\end{array}$ & $\begin{array}{l}\text { Oral } \\
\text { Ibuprofen, } \\
\text { glucosamine } \\
\text { sulphate or } \\
\text { placebo }\end{array}$ & $\begin{array}{l}1,200 \mathrm{mg} / \mathrm{d} \\
22 \mathrm{~d}\end{array}$ & $\begin{array}{l}(0-9 \\
\mathrm{cm})\end{array}$ & $\begin{array}{l}\text { BEx; AEx (15, } \\
16 \text { and } 22 \\
\text { days) }\end{array}$ & \multicolumn{2}{|c|}{$\begin{array}{l}\text { Not significant } \\
\text { "Ibuprofen is not capable of } \\
\text { inhibiting experimentally } \\
\text { induced muscle } \\
\text { tenderness/soreness" }\end{array}$} \\
\hline $\begin{array}{l}\text { Bourgeois } \\
\text { et al., } 1999\end{array}$ & $\begin{array}{l}\text { Cross- } \\
\text { over }\end{array}$ & $\begin{array}{l}\mathrm{n}=8 \\
8 \text { men } \\
\text { training level: } \\
\text { "moderately } \\
\text { trained" } \\
21.8 \pm 2.2 \text { yrs }\end{array}$ & $\begin{array}{l}\text { Unilateral knee } \\
\text { concentric/ } \\
\text { eccentric } \\
\text { weightlifting } \\
\text { with } 6 \text { sets x } \\
10 \text { repetitions } \\
\text { at } 80-85 \% \text { of } \\
\text { the } 1 \mathrm{RM} \\
\text { contraction }\end{array}$ & $\begin{array}{l}\text { Oral } \\
\text { naproxen or } \\
\text { placebo }\end{array}$ & $\begin{array}{l}1,000 \mathrm{mg} / \mathrm{d} \\
2 \mathrm{~d}\end{array}$ & \multicolumn{2}{|r|}{$\begin{array}{l}\text { VAS } \\
(0-10 \mathrm{~cm})\end{array}$} & $\begin{array}{l}\text { BEx; AEx (0, } 24 \text { and } 48 \\
\text { h) }\end{array}$ & $\begin{array}{l}\text { Not sigr } \\
\text { "NSAID } \\
\text { adminis } \\
\text { did not } \\
\text { muscle }\end{array}$ \\
\hline $\begin{array}{l}\text { Cannavino } \\
\text { et al., } 2003\end{array}$ & $\begin{array}{l}\text { Parallel } \\
\text { groups }\end{array}$ & $\begin{array}{l}\mathrm{n}=32 \\
32 \text { men } \\
\text { training level: } \\
\text { DNR } \\
18-35 \text { yrs }\end{array}$ & $\begin{array}{l}\text { Leg extension } \\
\text { and flexion } \\
\text { exercise } \\
\text { program } \\
\text { designed to } \\
\text { create DOMS } \\
\text { in quadriceps } \\
\text { muscles }\end{array}$ & $\begin{array}{l}\text { Topic } \\
\text { ketoprofen or } \\
\text { placebo }\end{array}$ & $\begin{array}{l}\text { cream } 10 \% \\
8 / 8 \mathrm{~h}\end{array}$ & \multicolumn{2}{|r|}{$(0-10 \mathrm{~cm})$} & $\begin{array}{l}\text { BEx; AEx (24 and } 48 \\
\text { h) }\end{array}$ & $\begin{array}{l}\text { Signific } \\
\text { "Transd } \\
\text { ketopro } \\
\text { appears } \\
\text { effectiv } \\
\text { reducin' } \\
\text { reportec }\end{array}$ \\
\hline $\begin{array}{l}\text { Croisier et } \\
\text { al., } 1996\end{array}$ & $\begin{array}{l}\text { Cross- } \\
\text { over }\end{array}$ & $\begin{array}{l}\mathrm{n}=10 \\
10 \text { men } \\
\text { training level: } \\
\text { "moderately } \\
\text { active" } \\
22.4 \pm 0.4 \text { yrs }\end{array}$ & $\begin{array}{l}\text { Eight stages of } \\
\text { five maximal } \\
\text { contractions of } \\
\text { the knee } \\
\text { extensor and } \\
\text { flexor muscle } \\
\text { groups of both } \\
\text { legs separated } \\
\text { by } 1 \text { min rest } \\
\text { phases, on a } \\
\text { Kin Trex device } \\
\text { at } 60^{\circ} / \mathrm{s} \\
\text { angular } \\
\text { velocity }\end{array}$ & $\begin{array}{l}\text { Oral } \\
\text { piroxicam or } \\
\text { placebo }\end{array}$ & $\begin{array}{l}20 \mathrm{mg} / \mathrm{d} \\
6 \mathrm{~d}\end{array}$ & & $\begin{array}{l}\text { VAS } \\
(0-10 \mathrm{~cm})\end{array}$ & $\begin{array}{l}\text { BEx; AEx (0, } 24 \text { and } 48 \\
\text { h) }\end{array}$ & $\begin{array}{l}\text { Not sigr } \\
\text { "Oral } \\
\text { adminis } \\
\text { of pirox } \\
\text { fails to } \\
\text { muscle } \\
\text { and DO } \\
\text { caused } \\
\text { strenuo } \\
\text { eccentri } \\
\text { exercis }\end{array}$ \\
\hline $\begin{array}{l}\text { Donnelly et } \\
\text { al., } 1988\end{array}$ & $\begin{array}{l}\text { Cross- } \\
\text { over }\end{array}$ & $\begin{array}{l}\mathrm{n}=20 \\
20 \text { men } \\
\text { training level: } \\
\text { "healthy } \\
\text { untrained" } \\
20 \pm 1 \text { yrs }\end{array}$ & $\begin{array}{l}\text { Running (heart } \\
\text { rate equivalent } \\
\text { to } 75 \% \text { of age } \\
\text { adjusted } \\
\text { maximum } 220 \text { - } \\
\text { age) for } 45 \\
\text { minutes. }\end{array}$ & $\begin{array}{l}\text { Oral } \\
\text { diclofenac or } \\
\text { placebo }\end{array}$ & $\begin{array}{l}150 \mathrm{md} / \mathrm{d} \\
(50 \mathrm{mg} \\
8 / 8 \mathrm{~h} ; 72 \mathrm{~h})\end{array}$ & & $\begin{array}{l}\text { VAS } \\
(1-10 \mathrm{~cm}) \\
\text { and pain } \\
\text { tolerance } \\
\text { threshold }\end{array}$ & $\begin{array}{l}\text { BEx and AEx }(6,24,48 \\
\text { and } 72 h)\end{array}$ & $\begin{array}{l}\text { Not sigr } \\
\text { “Diclof } \epsilon \\
\text { not infli } \\
\text { muscle } \\
\text { but mas } \\
\text { reduce } 1 \\
\text { associa } \\
\text { sorenes }\end{array}$ \\
\hline $\begin{array}{l}\text { Donnelly et } \\
\text { al., } 1990\end{array}$ & $\begin{array}{l}\text { Cross- } \\
\text { over }\end{array}$ & $\begin{array}{l}\mathrm{n}=32 \\
32 \text { men } \\
\text { training level: } \\
\text { "healthy } \\
\text { untrained" } \\
18 \text { - } 30 \text { yrs }\end{array}$ & $\begin{array}{l}\text { Running (heart } \\
\text { rate equivalent } \\
\text { to } 75 \% \text { of age } \\
\text { adjusted } \\
\text { maximum } 220- \\
\text { age) for } 45 \\
\text { minutes. }\end{array}$ & $\begin{array}{l}\text { Oral } \\
\text { ibuprofen or } \\
\text { placebo }\end{array}$ & $\begin{array}{l}2,400 \mathrm{mg} / \mathrm{d} \\
(600 \mathrm{mg} \\
6 / 6 \mathrm{~h} ; 72 \mathrm{~h})\end{array}$ & & $\begin{array}{l}\text { VAS } \\
(1-10 \mathrm{~cm}) \\
\text { and pain } \\
\text { tolerance } \\
\text { threshold }\end{array}$ & $\begin{array}{l}\text { BEx and AEx }(6,24,48 \\
\text { and } 72 h)\end{array}$ & $\begin{array}{l}\text { Not sigr } \\
\text { “Ibuprot } \\
\text { an appr } \\
\text { treatme } \\
\text { delayed } \\
\text { muscle } \\
\text { sorenes }\end{array}$ \\
\hline $\begin{array}{l}\text { Dudley et } \\
\text { al., } 1997\end{array}$ & $\begin{array}{l}\text { Cross- } \\
\text { over }\end{array}$ & $\begin{array}{l}\mathrm{n}=8 \\
8 \text { men } \\
\text { training level: } \\
\text { "young adult, } \\
\text { healthy" } \\
\text { age: DNR }\end{array}$ & $\begin{array}{l}\text { Ten sets of } \\
\text { seven to } 10 \\
\text { eccentric } \\
\text { actions with } \\
\text { each } \\
\text { quadriceps } \\
\text { femoris with a } \\
\text { load equal to } \\
85 \% \text { of the } \\
\text { eccentric one } \\
\text { repetition }\end{array}$ & $\begin{array}{l}\text { Oral } \\
\text { Naproxen or } \\
\text { placebo }\end{array}$ & $\begin{array}{l}600 \mathrm{mg} / \mathrm{d} \\
(200 \mathrm{mg} \\
8 / 8 \mathrm{~h} ; 4 \mathrm{~d})\end{array}$ & & $\begin{array}{l}\text { VAS } \\
(1-100 \mathrm{~mm})\end{array}$ & $\begin{array}{l}\text { BEx and AEx }(24,96 \\
\text { and } 240 \mathrm{~h})\end{array}$ & $\begin{array}{l}\text { Signific } \\
\text { "Napros } \\
\text { sodium } \\
\text { improve } \\
\text { recover } \\
\text { eccentri } \\
\text { biased } \\
\text { probabl } \\
\text { attenua } \\
\text { express } \\
\text { inflamn }\end{array}$ \\
\hline
\end{tabular}




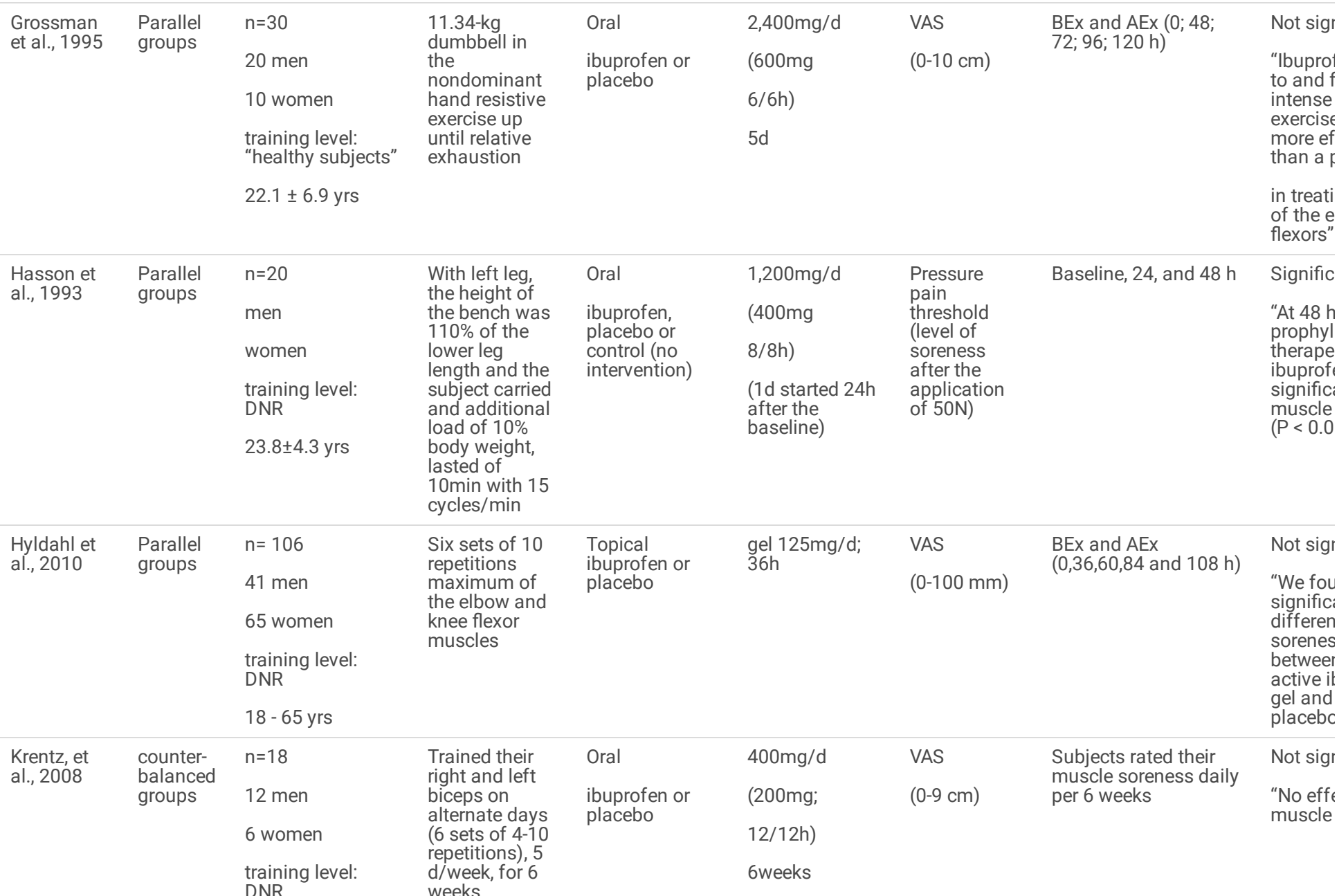

$24.1 \pm 0.6$ yrs

\begin{tabular}{|c|c|c|c|c|c|c|c|c|}
\hline $\begin{array}{l}\text { Lecomte et } \\
\text { al., } 1998\end{array}$ & $\begin{array}{l}\text { Cross- } \\
\text { over }\end{array}$ & $\begin{array}{l}n=20 \\
20 \text { men } \\
\text { training level: } \\
\text { DNR } \\
24.0 \pm 3.5 \text { yrs }\end{array}$ & $\begin{array}{l}\text { Eccentric } \\
\text { single-leg } \\
\text { exercises were } \\
\text { performed on } \\
\text { days } 1,3 \text {, and } \\
4 \text { to induce } \\
\text { DOMS in the } \\
\text { quadriceps } \\
\text { muscles (6 to } \\
15 \text { repetitions } \\
\text { maximum) }\end{array}$ & $\begin{array}{l}\text { Oral } \\
\text { naproxen or } \\
\text { placebo }\end{array}$ & $\begin{array}{l}1 \mathrm{~g} / \mathrm{d} \\
(500 \mathrm{mg} ; \\
12 / 12 \mathrm{~h}) \\
8 \mathrm{~d}\end{array}$ & $\begin{array}{l}\text { VAS } \\
(0-10 \mathrm{~cm})\end{array}$ & $\begin{array}{l}\text { Perception of muscle } \\
\text { soreness was } \\
\text { evaluated daily } \\
\text { throughout each } \\
\text { phase }\end{array}$ & $\begin{array}{l}\text { Signific } \\
\text { “Napro> } \\
\text { reducec } \\
\text { percept } \\
\text { sorenes } \\
\text { 3, when } \\
\text { sorenes } \\
\text { highest } \\
p=0.04 \text { ) }\end{array}$ \\
\hline $\begin{array}{l}\text { Loram et } \\
\text { al., } 2005\end{array}$ & $\begin{array}{l}\text { Cross- } \\
\text { over }\end{array}$ & $\begin{array}{l}\mathrm{n}=15 \\
10 \text { men } \\
5 \text { women } \\
\text { training level: } \\
\text { "physically active } \\
\text { but not } \\
\text { competitive" } \\
24.0 \pm 4.5 \text { yrs }\end{array}$ & $\begin{array}{l}\text { Downhill } \\
\text { running for } 30 \\
\text { min at a } 12 \% \\
\text { decline and a } \\
\text { speed of } 9 \\
\mathrm{~km} / \mathrm{h}\end{array}$ & $\begin{array}{l}\text { Oral } \\
\text { rofecoxib; } \\
\text { tramadol or } \\
\text { placebo }\end{array}$ & $\begin{array}{l}\text { rofecoxib } \\
50 \mathrm{mg} / \mathrm{d} \\
\text { Once a day } \\
4 \mathrm{~d} \\
\text { tramadol } \\
150 \mathrm{mg} / \mathrm{d} \\
(50 \mathrm{mg} / \mathrm{d} ; 8 / 8 \mathrm{~h}) \\
4 \mathrm{~d}\end{array}$ & $\begin{array}{l}\text { VAS } \\
(0-100 \mathrm{~mm}) \\
\text { and pressure } \\
\text { pain } \\
\text { threshold }\end{array}$ & $\begin{array}{l}\text { BEx and AEx ( } 24 \text { and } \\
72 \mathrm{~h})\end{array}$ & $\begin{array}{l}\text { Not sigr } \\
\text { "Muscle } \\
\text { sorenes } \\
\text { not aff } \epsilon \\
\text { significi } \\
\text { eitherd }\end{array}$ \\
\hline $\begin{array}{l}\text { McAnulty } \\
\text { et al., } 2007\end{array}$ & $\begin{array}{l}\text { Parallel } \\
\text { groups }\end{array}$ & $\begin{array}{l}n=60 \\
45 \text { men } \\
15 \text { women } \\
\text { training level: } \\
\text { "experienced } \\
\text { ultramarathoners" }\end{array}$ & $\begin{array}{l}160 \mathrm{~km} \\
\text { following the } \\
\text { Western States } \\
\text { Endurance Run }\end{array}$ & $\begin{array}{l}\text { Oral or topical } \\
\text { route not clear } \\
\text { in methodology } \\
\text { "Categorized } \\
\text { as NSAID users } \\
\text { if reported use } \\
\text { during running } \\
\text { and non-users } \\
\text { reported to } \\
\text { avoid NSAIDs" }\end{array}$ & $\begin{array}{l}\text { The ingested } \\
\text { doses were } \\
\text { performed } \\
\text { individually, as } \\
\text { performed } \\
\text { routinely by the } \\
\text { participants. }\end{array}$ & $\begin{array}{l}\text { VAS } \\
(0-10 \mathrm{~cm})\end{array}$ & $\begin{array}{l}\text { BEx and AEx } \\
(24,48,72,96,120,148 \\
\text { and } 172 h)\end{array}$ & 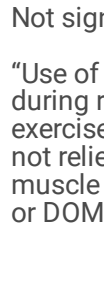 \\
\hline
\end{tabular}

Page $7 / 16$ 
$45.3 \pm 1.1$ yrs

\begin{tabular}{|c|c|c|c|c|c|c|c|c|}
\hline $\begin{array}{l}\text { Nieman et } \\
\text { al., } 2006\end{array}$ & $\begin{array}{l}\text { Parallel } \\
\text { groups }\end{array}$ & $\begin{array}{l}n=29 \\
29 \text { men } \\
\text { training level: } \\
\text { ultramarathoners } \\
47.9 \pm 1.4 \text { yrs }\end{array}$ & $\begin{array}{l}\text { Competing in a } \\
160-\mathrm{km} \text { race }\end{array}$ & $\begin{array}{l}\text { Oral } \\
\text { ibuprofen or } \\
\text { control (no } \\
\text { intervention) }\end{array}$ & $\begin{array}{l}600 \mathrm{mg} / \mathrm{d} \\
\text { And } \\
1,200 \mathrm{mg} / \mathrm{d} \\
\text { the day before } \\
\text { and on race } \\
\text { day, } \\
\text { respectively }\end{array}$ & $\begin{array}{l}\text { VAS } \\
(1-10 \mathrm{~cm})\end{array}$ & $\begin{array}{l}\text { BEx and after AEx } \\
(24,48,72,96,120,148 \\
\text { and } 172 \mathrm{~h})\end{array}$ & $\begin{array}{l}\text { Not sigr } \\
\text { "Ibuproł } \\
\text { compar } \\
\text { nonuse } \\
\text { athletes } \\
\text { compet } \\
\text { 160-km } \\
\text { not alte } \\
\text { sorenes }\end{array}$ \\
\hline $\begin{array}{l}\text { Rahnama } \\
\text { et al., } 2005\end{array}$ & $\begin{array}{l}\text { Parallel } \\
\text { groups }\end{array}$ & $\begin{array}{l}\mathrm{n}=44 \\
44 \text { men } \\
\text { training level: } \\
\text { "non-athletic" } \\
24.3 \pm 2.4 \text { yrs }\end{array}$ & $\begin{array}{l}70 \text { eccentric } \\
\text { contractions of } \\
\text { the biceps } \\
\text { muscle of the } \\
\text { non-dominant. } \\
\text { Set of } 10 \\
\text { contractions, } \\
\text { with load was } \\
80 \% \text { of the } \\
\text { maximal } \\
\text { voluntary } \\
\text { contraction. }\end{array}$ & $\begin{array}{l}\text { Oral } \\
\text { ibuprofen or } \\
\text { control (no } \\
\text { intervention) }\end{array}$ & $\begin{array}{l}2,800 \mathrm{mg} \\
1 \mathrm{~h} \text { before the } \\
\text { eccentric } \\
\text { actions up to } \\
48 \mathrm{~h} \text { after it }\end{array}$ & $\begin{array}{l}\text { VAS } \\
(1-30 \mathrm{~cm})\end{array}$ & $\begin{array}{l}\text { BEx and after AEx (1, } \\
24 \text { and } 48 \text { h) }\end{array}$ & $\begin{array}{l}\text { Signific } \\
\text { At } 24 \text { ar } \\
\text { greater } \\
(\mathrm{P}<0.0 \\
\text { observe } \\
\text { control }\end{array}$ \\
\hline $\begin{array}{l}\text { Rother et } \\
\text { al., } 2014\end{array}$ & $\begin{array}{l}\text { Cross- } \\
\text { over }\end{array}$ & $\begin{array}{l}\mathrm{n}=48 \\
25 \text { men } \\
23 \text { women } \\
\text { training level: } \\
\text { "health and had } \\
\text { an BMl > } 20 \text { and } \\
<30 \text { " }\end{array}$ & $\begin{array}{l}\text { Eccentric } \\
\text { exercise at } 45 \\
\% \text { of peak } \\
\text { torque until } \\
\text { volitional } \\
\text { fatigue }\end{array}$ & $\begin{array}{l}\text { Oral } \\
\text { etoricoxib or } \\
\text { placebo }\end{array}$ & $\begin{array}{l}90 \mathrm{mg} / \mathrm{d} \\
7 \mathrm{~d}\end{array}$ & $\begin{array}{l}\text { VAS } \\
(0-10 \mathrm{~cm})\end{array}$ & $\begin{array}{l}\text { BEx and AEx } \\
(24,48,72,96,120,148 \\
\text { and } 172 \mathrm{~h})\end{array}$ & $\begin{array}{l}\text { Not sigr } \\
\text { "Etorico } \\
\text { to show } \\
\text { significi } \\
\text { treatme } \\
\text { on pain" }\end{array}$ \\
\hline
\end{tabular}

Group 1 young

(18-40 yrs)

Group 2 elderly

(50-70 yrs)

\begin{tabular}{|c|c|c|c|c|c|c|c|c|}
\hline \multirow[t]{2}{*}{$\begin{array}{l}\text { Seidel et } \\
\text { al., } 2016\end{array}$} & $\begin{array}{l}\text { Parallel } \\
\text { groups }\end{array}$ & $\begin{array}{l}n=168 \\
86 \text { men } \\
82 \text { women } \\
\text { training level: } \\
\text { "Healthy" } \\
18-55 \text { yrs }\end{array}$ & $\begin{array}{l}\text { Walked for } \\
\text { approximately } \\
40 \text { min } \\
\text { downstairs } \\
\text { with a total } \\
\text { altitude of } \\
300-400 \mathrm{~m}\end{array}$ & $\begin{array}{l}\text { Topical } \\
\text { ketoprofen }+ \\
\text { oral placebo } \\
\text { (two groups); } \\
\text { Oral ketoprofen } \\
\text { or oral placebo } \\
\text { (two groups) }\end{array}$ & $\begin{array}{l}\text { Topical } \\
\text { Group1: 100mg } \\
\text { ketoprofen + } \\
\text { oral placebo. } \\
\text { Group2: 200mg } \\
\text { ketoprofen+ } \\
\text { oral placebo } \\
\\
\text { Oral } \\
\text { Group 1: } \\
\text { 100mg oral } \\
\text { ketoprofen + } \\
\text { topical placebo } \\
\\
\text { Group 2: } \\
\text { placebo } \\
\text { capsule + } \\
\text { topical } \\
\text { ketoprofen }\end{array}$ & $\begin{array}{l}\text { VAS } \\
(0-9 \mathrm{~cm})\end{array}$ & $\begin{array}{l}\text { BEx and AEx }(24,48, \\
96,192 \text { and } 288 \mathrm{~h})\end{array}$ & $\begin{array}{l}\text { Not sigr } \\
\text { "Gel anı } \\
\text { were su } \\
\text { oral ket } \\
\text { in reduc } \\
\text { muscle } \\
\text { ness fo } \\
\text { exercisє } \\
\text { Furtherr } \\
\text { ketopro } \\
\text { delayed } \\
\text { from m } \\
\text { sorenes }\end{array}$ \\
\hline & & & & & $\begin{array}{l}12 / 12 \mathrm{~h} \\
7 \mathrm{~d}\end{array}$ & & & \\
\hline $\begin{array}{l}\text { Simmons } \\
\text { et al., } 2018\end{array}$ & $\begin{array}{l}\text { Parallel } \\
\text { groups }\end{array}$ & $\begin{array}{l}\mathrm{n}=37 \\
\text { training level: } \\
\text { DNR } \\
\text { age: DNR }\end{array}$ & $\begin{array}{l}\text { Exercise } \\
\text { regimen and } \\
\text { utilizing a } \\
\text { customized, } \\
\text { non-invasive } \\
\text { armband } \\
\text { (Band- } 0^{T M} \text {, }\end{array}$ & $\begin{array}{l}\text { Oral } \\
\text { ibuprofen or } \\
\text { placebo }\end{array}$ & $\begin{array}{l}1600 \mathrm{mg} / \mathrm{d} \\
(400 \mathrm{mg} \\
4 \text { doses }) \\
1 \mathrm{~d}\end{array}$ & $\begin{array}{l}\text { VAS } \\
\text { (0-10 cm); } \\
\text { sum of Pain } \\
\text { Intensity } \\
\text { Differences } \\
\text { (SPID); and } \\
\text { sum of }\end{array}$ & $\begin{array}{l}\text { BEx and AEx }(0,24 \\
\text { and } 48 \mathrm{~h})\end{array}$ & $\begin{array}{l}\text { Signific } \\
\text { "Ibuprol } \\
\text { safe an } \\
\text { effectiv } \\
\text { DOMS | }\end{array}$ \\
\hline
\end{tabular}




\begin{tabular}{|c|c|c|c|c|c|c|c|c|}
\hline & & & $\begin{array}{l}\text { patent } \\
\text { pending) }\end{array}$ & & & $\begin{array}{l}\text { Stiffness } \\
\text { Movement } \\
\text { Differences } \\
\text { (SSMD) }\end{array}$ & & \\
\hline \multirow{5}{*}{$\begin{array}{l}\text { Singla et } \\
\text { al., } 2015\end{array}$} & \multirow{5}{*}{$\begin{array}{l}\text { Parallel } \\
\text { groups }\end{array}$} & $n=24$ & \multirow{5}{*}{$\begin{array}{l}\text { Two sets with } \\
\text { approximately } \\
10-20 \text { Ibs of } \\
\text { weight with } \\
\text { maximum } \\
\text { tolerated } \\
\text { weight (MTW) } \\
\text { on the leg curl } \\
\text { machine }\end{array}$} & \multirow{5}{*}{$\begin{array}{l}\text { Topical } \\
\text { Diclofenac or } \\
\text { placebo }\end{array}$} & \multirow{5}{*}{$\begin{array}{l}\text { Diclofenac } \\
\text { gel 1\% } \\
\text { (DSG 1\%; 48h) }\end{array}$} & \multirow{5}{*}{$\begin{array}{l}\text { VAS } \\
(0-10 \mathrm{~cm})\end{array}$} & \multirow{5}{*}{$\begin{array}{l}\text { BEx and AEx } \\
(24,48,72,96,120,148 \\
\text { and } 172 \mathrm{~h})\end{array}$} & Signific \\
\hline & & 15 men & & & & & & "The stı \\
\hline & & 9 women & & & & & & analges \\
\hline & & $\begin{array}{l}\text { training level: } \\
\text { "healthy volunteer } \\
\text { subjects" }\end{array}$ & & & & & & $\begin{array}{l}\text { topical } \\
\text { over pla } \\
\text { subject: } \\
\text { experier }\end{array}$ \\
\hline & & $28+3.5$ yrs & & & & & & DOMS" \\
\hline \multirow{9}{*}{$\begin{array}{l}\text { Smith et } \\
\text { al., } 1995\end{array}$} & \multirow{9}{*}{$\begin{array}{l}\text { Parallel } \\
\text { groups }\end{array}$} & $n=36$ & \multirow{9}{*}{$\begin{array}{l}\text { The eccentric } \\
\text { phase of a } \\
\text { supine bench } \\
\text { press at a } \\
\text { resistance } \\
\text { equivalent to } \\
120 \% \text { of } \\
\text { maximum } \\
\text { concentric } \\
\text { strength, } 1 \text { RM } \\
\text { ( } 4 \text { sets, } 12 \\
\text { repetitions/set) }\end{array}$} & \multirow{9}{*}{$\begin{array}{l}\text { Oral } \\
\text { aspirin, } \\
\text { acetaminophen } \\
\text { or } \\
\text { placebo }\end{array}$} & Aspirin & \multirow{9}{*}{$\begin{array}{l}\text { VAS } \\
(1-10 \mathrm{~cm})\end{array}$} & \multirow{9}{*}{$\begin{array}{l}\text { BEx and AEx } \\
(24,48,72,96 \text { and } 120 \\
\text { h) }\end{array}$} & Not sigr \\
\hline & & 36 men & & & $3.0 \mathrm{~g} / \mathrm{d}$ & & & "These \\
\hline & & training level: & & & (750mg & & & adminis \\
\hline & & untrained" & & & 6/6h) & & & acetam \\
\hline & & \multirow{5}{*}{$24.4 \pm 1.5 \mathrm{yrs}$} & & & & & & the DON \\
\hline & & & & & Acetoaminophe & & & \\
\hline & & & & & $(3.0 \mathrm{~g} / \mathrm{d}$ & & & \\
\hline & & & & & $750 \mathrm{mg} ; 6 / 6 \mathrm{~h})$ & & & \\
\hline & & & & & $5 d$ & & & \\
\hline \multirow{9}{*}{$\begin{array}{l}\text { Stone et al., } \\
2002\end{array}$} & \multirow{9}{*}{$\begin{array}{l}\text { Parallel } \\
\text { groups }\end{array}$} & $\mathrm{n}=40$ & \multirow{9}{*}{$\begin{array}{l}30 \text { repetitions } \\
\text { with } \\
\text { the } 2.27-\mathrm{kg} \\
\text { dumbbell }\end{array}$} & \multirow{9}{*}{$\begin{array}{l}\text { Oral } \\
\text { ibuprofen, } \\
\text { bromelain, } \\
\text { placebo or } \\
\text { control (no } \\
\text { intervention) }\end{array}$} & Bromelain & \multirow{9}{*}{$\begin{array}{l}\text { VAS } \\
(1-10 \mathrm{~cm})\end{array}$} & \multirow{9}{*}{$\begin{array}{l}\text { BEx and AEx }(24,48,72 \\
\text { and } 96 \mathrm{~h})\end{array}$} & Not sigr \\
\hline & & \multirow{3}{*}{$\begin{array}{l}20 \text { men } \\
20 \text { women } \\
\text { training level: } \\
\text { DNR }\end{array}$} & & & $900 \mathrm{mg} / \mathrm{d}$ & & & \multirow{8}{*}{$\begin{array}{l}\text { "No diff } \\
\text { among } \\
\text { treatme } \\
\text { observe }\end{array}$} \\
\hline & & & & & $\begin{array}{l}\text { (300 mg; } \\
8 / 8 \text { hours) }\end{array}$ & & & \\
\hline & & & & & 3 days; & & & \\
\hline & & $23 \pm 3.2 \mathrm{yrs}$ & & & & & & \\
\hline & & & & & Ibuprofen & & & \\
\hline & & & & & $1,200 \mathrm{mg} / \mathrm{d}$ & & & \\
\hline & & & & & (400mg; 8/8h) & & & \\
\hline & & & & & $3 d$ & & & \\
\hline \multirow{8}{*}{$\begin{array}{l}\text { Svensson } \\
\text { et al., } 1997\end{array}$} & \multirow{8}{*}{$\begin{array}{l}\text { Parallel } \\
\text { groups }\end{array}$} & $\mathrm{n}=10$ & \multirow{8}{*}{$\begin{array}{l}\text { six 5-minute } \\
\text { bouts of } \\
\text { submaximal } \\
\text { eccentric jaw } \\
\text { exercise }\end{array}$} & Topical & Oral & Pain & BEx and AEx $(24,48$ & Signific \\
\hline & & 10 men & & Fin, & $1,200 \mathrm{mg} / \mathrm{d}$ & threshold & & "Nonst $\epsilon$ \\
\hline & & $\begin{array}{l}\text { training level: } \\
\text { DNR }\end{array}$ & & Oral & (400mg; 8/8h) & & & $\begin{array}{l}\text { inflamn } \\
\text { associa }\end{array}$ \\
\hline & & & & $\begin{array}{l}\text { ibuprofen or } \\
\text { placebo }\end{array}$ & $3 d$ & & & $\begin{array}{l}\text { significi } \\
\text { higher- } \\
\text { pain thr }\end{array}$ \\
\hline & & & & & Topical & & & with \\
\hline & & & & & $2 g(5 \%)$ & & & topical : \\
\hline & & & & & $8 / 8 h$ & & & $\begin{array}{l}<.05) a \\
\text { placebc }\end{array}$ \\
\hline & & & & & $3 d$ & & & $.05)^{\prime \prime}$ \\
\hline $\begin{array}{l}\text { Tokmakidis } \\
\text { et al. 2003 }\end{array}$ & $\begin{array}{l}\text { Parallel } \\
\text { groups }\end{array}$ & $\mathrm{n}=19$ & $\begin{array}{l}\text { Six sets of } 10 \\
\text { eccentric }\end{array}$ & Oral & $1,200 \mathrm{mg} / \mathrm{d}$ & VAS & $\begin{array}{l}\text { BEx and AEx }(4,6,24 \\
\text { and } 48 \mathrm{~h})\end{array}$ & Signific \\
\hline & & 14 men & $\begin{array}{l}\text { actions with a } \\
\text { resistance of }\end{array}$ & ibuprofen or & (400mg; 8/8h) & $(1-10 \mathrm{~cm})$ & & $\begin{array}{l}\text { “Ibupro1 } \\
\text { vielded }\end{array}$ \\
\hline & & 5 women & $\begin{array}{l}100 \% \text { of the } \\
\text { maximal }\end{array}$ & & $2 d$ & & & significi \\
\hline & & & $\begin{array}{l}\text { concentric } \\
\text { strength (1RM) }\end{array}$ & & & & & $\begin{array}{l}0.05) \text { at } \\
\text { hours" }\end{array}$ \\
\hline & & $24.6 \pm 3$ yrs & & & & & & \\
\hline Trappe et & Parallel & $n=24$ & 10-14 sets of & Oral & Ibuprofen & VAS & BEx and AEx $(0,24$ & Not sigr \\
\hline & & 24 men & $\begin{array}{l}\text { repetitions at } \\
120 \% \text { of }\end{array}$ & $\begin{array}{l}\text { ibuprofen; } \\
\text { acetaminophen }\end{array}$ & $1,200 \mathrm{mg} / \mathrm{d}$ & $\begin{array}{l}(1-9 \mathrm{~cm}) \text { and } \\
\text { level of }\end{array}$ & & "No efft \\
\hline & & $\begin{array}{l}\text { training level: } \\
\text { DNR }\end{array}$ & $\begin{array}{l}\text { concentric one- } \\
\text { repetition } \\
\text { maximum with }\end{array}$ & or placebo & $\begin{array}{l}\text { (400mg } \\
\text { three doses) }\end{array}$ & $\begin{array}{l}\text { soreness } \\
\text { after the }\end{array}$ & & sorenes \\
\hline
\end{tabular}

Page 9/16 


\begin{tabular}{|c|c|c|c|c|c|c|c|c|}
\hline & & $25 \pm 3$ yrs & $\begin{array}{l}\text { the knee } \\
\text { extensors }\end{array}$ & & $1 \mathrm{~d}$ & $\begin{array}{l}\text { application } \\
\text { of } 40 \mathrm{~N}\end{array}$ & & \\
\hline & & & & & $\begin{array}{l}\text { Acetaminophen } \\
4000 \mathrm{mg} / \mathrm{d} \\
8 / 8 \mathrm{~h} \\
1^{\text {st }} \cdot \text { dose one } \\
1,5002^{\text {nd }} \cdot \text { dose } \\
1,500 \mathrm{mg}, 3^{\text {rd }} \text {. } \\
\text { dose } 1,000 \mathrm{mg} \\
1 \mathrm{~d}\end{array}$ & & & \\
\hline $\begin{array}{l}\text { Vella et al., } \\
2016\end{array}$ & $\begin{array}{l}\text { Parallel } \\
\text { groups }\end{array}$ & $\begin{array}{l}\mathrm{n}=16 \\
16 \text { men } \\
\text { training level: } \\
\text { “healthy subjects” } \\
23.9 \pm 1.3 \text { yrs }\end{array}$ & $\begin{array}{l}\text { three sets of } \\
8-10 \\
\text { repetitions } \\
\text { performed on a } \\
\text { Smith machine } \\
\text { assisted squat, } \\
\text { a } 45^{\circ} \text { leg press } \\
\text { and a leg } \\
\text { extension at } \\
80 \% \text { of a } \\
\text { predicted } 1 \mathrm{RM}\end{array}$ & $\begin{array}{l}\text { Oral } \\
\text { ibuprofen or } \\
\text { placebo }\end{array}$ & $\begin{array}{l}1,200 \mathrm{mg} / \mathrm{d} \\
\text { (400mg } \\
\text { three doses) } \\
\text { First dose } \\
\text { immediately } \\
\text { prior to the first } \\
\text { muscle biopsy } \\
\text { two doses at } 6 \\
\text { and } 12 \mathrm{~h} \\
\text { following the } \\
\text { exercise } \\
\text { protocols. }\end{array}$ & $\begin{array}{l}\text { VAS } \\
(1-10 \mathrm{~cm})\end{array}$ & $\begin{array}{l}\text { BEx and AEx (0 and } \\
24 \mathrm{~h})\end{array}$ & $\begin{array}{l}\text { Not sigr } \\
\text { “No effe } \\
\text { muscle } \\
\text { sorenes }\end{array}$ \\
\hline
\end{tabular}

Legend: $y r s=y e a r s ; ~ n=$ number of participants; $D O M S=$ Delayed Onset Muscle Soreness; RM= maximum repetition; VAS= Visual analog scale; $D$ NR : $\iota$ $\mathrm{d}=$ days; $\mathrm{h}=$ hours; $\mathrm{mg}=$ =milligrams; $\mathrm{BEx}=$ Before exercise; $\mathrm{AEx}=$ After exercise; $\mathrm{N}$ = newton; The characterization of the studies, subjects and protocols exactly using the paper's authors description. ${ }^{*}$ written exactly as stated in the article

\section{Effect of NSAIDs to treat DOMS}

In order to assess the significance in the described use of NSAIDs on DOMS we evaluate the studies using the Random-Effect model $\left(I^{2}=93 \%\right)$. Our analyzes showed no difference regarding the attributed use of NSAIDs (21 studies, $n=955 ; \mathrm{SMD}=0.02 ; 95 \% \mathrm{Cl}-0.58,0.63 ; \mathrm{p}=0.94 ; \mathrm{I}=93 \%)$ Figure 3 .

Figure 3. Forest plot showing the effects of NSAIDs (experimental) versus control condition on the management of DOMS. SD: standard deviation; Std: standardized; Cl: confidence interval. Program: (RevMan, 5.3.5); heterogeneity: $\mathrm{Tau}^{2}=1.59 ; \mathrm{Ch}^{2}=2269,77, \mathrm{df}=19(\mathrm{P} 0.000001) ; \mathrm{I}^{2}=93 \%$

\section{Discussion}

Either Inflammation or pain can be limiting factor for training and exercise and the NSAIDs are widely used in the handling of both symptoms. These drugs are broadly spread either following medical prescription or in an over-the-counter use [41]. In this study, we analyzed by the way of meta-analyze studies related to the effectiveness of selective and non-selective NSAIDs in the management of DOMS related to exercise.

We analyzed by not limiting specific characteristics. This method allowed a holistic perception regarding the analyzes, related to different dose responses, NSAIDs and population profiles. The mechanisms and relationship between DOMS and inflammation was previously described [9]. And there is current evidence showing improvement in pain and inflammatory processes in response to the use of these drugs $[28,35,38,39]$. While, additional studies showed 
that the use of NSAIDs is related to the inhibition of satellite cells, negatively influencing the development of healing, adaptation to stress and subsequently muscle regeneration $[42,43]$.

There is contradiction in the literature about the functional effects of NSAIDs in signaling and muscle regeneration. Mackey et al. (2016) evaluated the effect of ibuprofen on satellite cells activity after eccentric contractions induced by electrical stimulus [44]. Their study showed that ibuprofen-treated subjects had increased levels of cell proliferation and faster repair of myofibrils. It is important to highlight that the use of electrical stimulation to induce muscle damage is a limiting factor of the study. Electrical-induced muscle contractions do not fully reflect physiological conditions of exercise [45]. Thus, it is important emphasize this limitation. Other studies showed no correlation in the effects of NSAIDs in the outcome, pain or functional limitation, of DOMS [7, 9, 32, 40]. A possible justification is an impairment in muscle regeneration capacity due to decreasing in monocytes differentiation followed by inhibition of the inflammatory process, and the change in cytokine's signaling. These effects together could be responsible for systemic responses of neuro-muscular adaptation and muscle regeneration $[4,12]$. In a practical context the weakening of the described functions tends to limit the subsequent performance in either training or competition [46].

NSAIDs are overused in clinical practice for the treatment of various conditions, including DOMS [41]. The studies by Paulsen et al. [12] and Schoenfeld et al. [4] suggest that mild clinical manifestations of DOMS do not require treatment with NSAIDs. Clinical trials using rofecoxib showed an exponential increase in acute myocardial infarction, justified by high levels of toxicity in selective cyclooxygenase inhibitors [15]. Also, NSAIDs inhibits prostanoids synthesis bringing adverse impacts including side effects on the gastrointestinal tract, renal and cardiovascular system [15] [47-50]. Such information is of concern and should be taken into consideration to evaluate the real need of NSAIDs use associated with the specific clinical condition of each patient [51]. Due to the adverse effects and functional impairment, the indiscriminate use of NSAIDs is alarming. This problem is aggravated by the its prolonged use, mostly without a medical prescription [15].

To the best of our knowledge this is the first systematic review and meta-analysis to investigate the effects of the use of NSAIDs in the treatment of DOMS. Our meta-analysis showed that the use of NSAIDs is neither superior nor responsible for significant levels of improvement when compared to the control/placebo situation. The importance of our findings for clinical practice lies in highlight important evidence about the ineffectiveness use of NSAIDs in DOMS and the possible hazards of its indiscriminate use. The current literature provides a variety of therapeutic options for the treatment of muscle pain [52] with reduced adverse effects and can be considered as an alternative resource whenever possible.

Our meta- analysis did not support the use of oral NSAIDs for the treatment of DOMS. Two articles using topical NSAIDs were selected in our meta-analysis, all of them with "good outcomes". It is difficult in guaranteeing a blind topical study since that some subjects can fell the presence of the active compound (ref). Another possible explanation is that the local drug concentration in topical use can be a reason for the best results comparing with the oral route (ref).

Diclofenac and aspirin are the world most used NSAIDs while ibuprofen or naproxen are far below (ref). During our review we found that ibuprofen was the must examined oral NSAIDs (52.2\%), followed by naproxen (13.0\%) of the studies. The less investigated drugs were either aspirin or diclofenac (4.3\% each). The majority of the studies (96.2\%) were conducted in countries with Very High Human Development Index (HDI) according to the United Nations Development Program (ref). We think that researchers and volunteers either propose or engage in studies according with their experiences and resources. This lack of original studies may present a bias in the available published papers leading to a limitation in the results to be analyzed. Our analyzes can be biased by these heterogenicity of original investigations. It is always important to emphasize that correlation it is not necessarily cause and effect. A more comprehensive experimental study in at least most used NSAIDs (in both oral and topical administration) should investigate their mechanisms of action in DOMS.

The majority of the 26 studies selected in this work, ( 92\%) used a visual analog scale (VAS) as a form of pain assessment to the subjects. VAS is a reliability and efficient tool for clinical research regarding pain [53]. However, VAS is an ordinal scale presented in numbers and should not be confused as a linear numeric scale. This misunderstand of the scale leads to an essential misconception in data analysis. While found in several scientific papers, it is not wise to convert subjective perceptions in numbers, mathematizing data for further statistical analyzes. Pain is a subjective symptom and its perception includes both psychological inputs and subject behavior [54]. Performing a meta-analysis with subjective data is always a challenge and a method limitation.

Some limitations inherent to the presented outcomes need to be reported. First of all, the majority of the protocols used in the included trials were unsatisfactory, which leads to inadequate evidence. The lack of consistency between the different methodologies of the studies compromised a homogeneous comparison and solid discussions. So, our results and discussion should be interpreted taking into consideration such circumstances. It needs to be emphasized that our findings are related to the use of different drugs and dose-response, as well as protocols for muscle damage, in the original investigations. Such facts should be considered and not extrapolated to different conditions than those reported in this study. Trying to analyze different small clinical studies with broad methodology is always a challenge and our goal was to reunite combined evidences that could enlighten the field.

\section{Conclusions}

This study provides evidence that the use of NSAIDs in the management of DOMS does not appear to be superior to the control condition and/or placebo. However, these interpretations should be analyzed with caution, since the types of NSAIDs, dose/response and volume/intensity of the effort made to induce different kind of muscle damage and, then different outputs. As continuous use can trigger several adverse effects in body systems, it is relevant that future studies demonstrate the real improvement prospects on the DOMS. 


\section{Declarations}

What is already known

Delayed Onset Muscle Soreness is a clinical physiological condition that limits subsequent performance levels. NSAIDs are world used to treat either inflammation or pain mostly without medical prescription.

\section{What are the new findings?}

There is no significant improvement in DOMS observed with the use of NSAID;

Different NSAIDs do not seem to give different clinical responses.

\section{ACKNOWLEDGEMENTS}

The authors would like to thank the corresponding authors of studies included in this review who answered to our requests providing further information on their published data.

\section{CONFLICT OF INTEREST STATEMENT}

All authors declare no conflict of interest.

\section{AUTHOR CONTRIBUTIONS}

RLN designed the study, conducted the analyzes, and wrote the manuscript.

JSSL and ASM assisted in the acquisition, analysis, and interpretation of data, reviewed and edited the article.

LCC made substantial contributions including conception; design of the study; writing and final revision of the manuscript.

All authors read and approved the final manuscript.

\section{References}

1. Mizumura, K. and T. Taguchi, Delayed onset muscle soreness: Involvement of neurotrophic factors. J Physiol Sci, 2016. 66(1): p. 43-52.

2. Agten, C.A., et al., Delayed-Onset Muscle Soreness: Temporal Assessment With Quantitative MRI and Shear-Wave Ultrasound Elastography. AJR Am J Roentgenol, 2017. 208(2): p. 402-412.

3. Ota, H., et al., TRPV1 and TRPV4 play pivotal roles in delayed onset muscle soreness. PLoS One, 2013. 8(6): p. e65751.

4. Schoenfeld, B.J., The use of nonsteroidal anti-inflammatory drugs for exercise-induced muscle damage: implications for skeletal muscle development. Sports Med, 2012. 42(12): p. 1017-28.

5. Markworth, J.F., et al., Ibuprofen treatment blunts early translational signaling responses in human skeletal muscle following resistance exercise. Journal of Applied Physiology, 2014. 117(1): p. 20-28.

6. Kim, J. and J. Lee, A review of nutritional intervention on delayed onset muscle soreness. Part I. J Exerc Rehabil, 2014. 10(6): p. 349-56.

7. Trappe, T.A., et al., Effect of ibuprofen and acetaminophen on postexercise muscle protein synthesis. Am J Physiol Endocrinol Metab, 2002. 282(3): p. E551-6.

8. Bourgeois, J., et al., Naproxen does not alter indices of muscle damage in resistance-exercise trained men. Med Sci Sports Exerc, 1999. 31(1): p. 4-9.

9. Vella, L., et al., Ibuprofen Ingestion Does Not Affect Markers of Post-exercise Muscle Inflammation. Front Physiol, 2016.7 : p. 86.

10. Singla, N., et al., Delayed-onset muscle soreness: a pilot study to assess analgesic study design features. Pain, 2015. 156(6): p. 1036-45.

11. Tokmakidis, S.P., et al., The effects of ibuprofen on delayed muscle soreness and muscular performance after eccentric exercise. J Strength Cond Res, 2003. 17(1): p. 53-9.

12. Paulsen, G., et al., Leucocytes, cytokines and satellite cells: what role do they play in muscle damage and regeneration following eccentric exercise? Exerc Immunol Rev, 2012. 18: p. 42-97.

13. Mackey, A.L., et al., The influence of anti-inflammatory medication on exercise-induced myogenic precursor cell responses in humans. J Appl Physiol (1985), 2007. 103(2): p. 425-31. 
14. Bassini, A. and L.C. Cameron, Sportomics: building a new concept in metabolic studies and exercise science. Biochem Biophys Res Commun, 2014. 445(4): p. 708-16.

15. Varga, Z., S.R.A. Sabzwari, and V. Vargova, Cardiovascular Risk of Nonsteroidal Anti-Inflammatory Drugs: An Under-Recognized Public Health Issue. Cureus, 2017. 9(4): p. e1144.

16. Lopes, J.S.S., et al., Training with elastic and conventional devices on body composition: systematic review and meta-analysis. Fisioterapia em Movimento, 2020. 33.

17. Lopes, J.S.S., et al., Effects of training with elastic resistance versus conventional resistance on muscular strength: A systematic review and metaanalysis. SAGE Open Med, 2019. 7: p. 2050312119831116.

18. Arendt-Nielsen, L., et al., A Double-Blind Randomized Placebo Controlled Parallel Group Study Evaluating the Effects of Ibuprofen and Glucosamine Sulfate on Exercise Induced Muscle Soreness. Journal of Musculoskeletal Pain, 2007. 15(1): p. 21-28.

19. Machado, A.F., et al., Phototherapy on Management of Creatine Kinase Activity in General Versus Localized Exercise: A Systematic Review and MetaAnalysis. Clin J Sport Med, 2020. 30(3): p. 267-274.

20. Henschke, N., et al., Behavioural treatment for chronic low-back pain. Cochrane Database Syst Rev, 2010(7): p. CD002014.

21. Smith, L.L., et al., Do over-the-counter analgesics reduce delayed onset muscle soreness and serum creatine kinase values? Sports Medicine, Training and Rehabilitation, 1995. 6(2): p. 81-88.

22. Grossman, J.M., et al., Effect of Ibuprofen Use on Delayed Onset Muscle Soreness of the El bow Flexors. 1995, Human Kinetics Publishers: Journal of Sport Rehabilitation. p. 253-263.

23. Hasson, S.M., et al., Effect of ibuprofen use on muscle soreness, damage, and performance: a preliminary investigation. Med Sci Sports Exerc, 1993. 25(1): p. 9-17.

24. McAnulty, S., et al., Effect of NSAID on muscle injury and oxidative stress. Int J Sports Med, 2007. 28(11): p. 909-15.

25. Hyldahl, R.D., et al., Effects of ibuprofen topical gel on muscle soreness. Med Sci Sports Exerc, 2010. 42(3): p. 614-21.

26. Dudley, G.A., et al., Efficacy of naproxen sodium for exercise-induced dysfunction muscle injury and soreness. Clin J Sport Med, 1997. 7(1): p. 3-10.

27. Cannavino, C.R., et al., Efficacy of transdermal ketoprofen for delayed onset muscle soreness. Clin J Sport Med, 2003. 13(4): p. 200-8.

28. Simmons, G., et al., Enhancing methods for the delayed onset muscle soreness (DOMS) pain model. The Journal of Pain, 2018. 19 (3): p. S81.

29. Nieman, D.C., et al., Ibuprofen use, endotoxemia, inflammation, and plasma cytokines during ultramarathon competition. Brain Behav Immun, 2006. 20(6): p. 578-84.

30. Stone, M.B., et al., Preliminary comparison of bromelain and Ibuprofen for delayed onset muscle soreness management. Clin J Sport Med, 2002. 12(6): p. 373-8.

31. Lecomte, J.M., V.J. Lacroix, and D.L. Montgomery, A randomized controlled trial of the effect of naproxen on delayed onset muscle soreness and muscle strength. Clin J Sport Med, 1998. 8(2): p. 82-7.

32. Krentz, J.R., et al., The effects of ibuprofen on muscle hypertrophy, strength, and soreness during resistance training. Appl Physiol Nutr Metab, 2008. 33(3): p. 470-5.

33. Donnelly, A.E., et al., Effects of a non-steroidal anti-inflammatory drug on delayed onset muscle soreness and indices of damage. Br $\mathrm{J}$ Sports Med, 1988. 22(1): p. 35-8.

34. Donnelly, A.E., R.J. Maughan, and P.H. Whiting, Effects of ibuprofen on exercise-induced muscle soreness and indices of muscle damage. Br J Sports Med, 1990. 24(3): p. 191-5.

35. Rahnama, N., F. Rahmani-Nia, and K. Ebrahim, The isolated and combined effects of selected physical activity and ibuprofen on delayed-onset muscle soreness. J Sports Sci, 2005. 23(8): p. 843-50.

36. Seidel, E.J., et al., A randomised trial comparing the efficacy and safety of topical ketoprofen in Transfersome(B) gel (IDEA-033) with oral ketoprofen and drug-free ultra-deformable Sequessome ${ }^{T M}$ vesicles (TDT 064) for the treatment of muscle soreness following exercise. J Sports Sci, 2016. 34(1): p. 88-95.

37. Matthias, R., S. Egbert Johannes, and K. Ilka Rother and Steffen, Randomized, Placebo Controlled Study of the Effects of Etoricoxib on Markers of Inflammation, Pain and Muscle Force After Eccentric Exercise. Clinical Anti-Inflammatory \& Anti-Allergy Drugs (Discontinued), 2014. 1(2): p. 99-110.

38. Svensson, P., L. Houe, and L. Arendt-Nielsen, Effect of systemic versus topical nonsteroidal anti-inflammatory drugs on postexercise jaw-muscle soreness: a placebo-controlled study. J Orofac Pain, 1997. 11(4): p. 353-62.

39. Croisier, J.L., et al., Piroxicam fails to reduce myocellular enzyme leakage and delayed onset muscle soreness induced by isokinetic eccentric exercise. Mediators Inflamm, 1996. 5(3): p. 230-4.

40. Loram, L.C., D. Mitchell, and A. Fuller, Rofecoxib and tramadol do not attenuate delayed-onset muscle soreness or ischaemic pain in human volunteers. Canadian Journal of Physiology and Pharmacology, 2005. 83(12): p. 1137-1145.

41. Hotfiel, T., et al., [Current Conservative Treatment and Management Strategies of Skeletal Muscle Injuries]. Z Orthop Unfall, 2016. 154(3): p. 245-53.

42. Järvinen, T.A., et al., Muscle injuries: biology and treatment. Am J Sports Med, 2005. 33(5): p. 745-64.

43. Sciorati, C., et al., Cell death, clearance and immunity in the skeletal muscle. Cell Death Differ, 2016. 23(6): p. 927-37.

44. Mackey, A.L., et al., Activation of satellite cells and the regeneration of human skeletal muscle are expedited by ingestion of nonsteroidal antiinflammatory medication. FASEB J, 2016. 30(6): p. 2266-81.

45. Backus, D., et al., Outcomes After Functional Electrical Stimulation Cycle Training in Individuals with Multiple Sclerosis Who Are Nonambulatory. Int J MS Care, 2017. 19(3): p. 113-121.

Page $13 / 16$ 
46. Toumi, H. and T.M. Best, The inflammatory response: friend or enemy for muscle injury? Br J Sports Med, 2003. 37(4): p. 284-6.

47. Bombardier, C., et al., Comparison of upper gastrointestinal toxicity of rofecoxib and naproxen in patients with rheumatoid arthritis. VIGOR Study Group. N Engl J Med, 2000. 343(21): p. 1520-8, 2 p following 1528.

48. García Rodríguez, L.A. and H. Jick, Risk of upper gastrointestinal bleeding and perforation associated with individual non-steroidal anti-inflammatory drugs. Lancet, 1994. 343(8900): p. 769-72.

49. MacDonald, T.M., et al., Association of upper gastrointestinal toxicity of non-steroidal anti-inflammatory drugs with continued exposure: cohort study. BMJ, 1997. 315(7119): p. 1333-7.

50. Palmer, R., et al., Effects of nabumetone, celecoxib, and ibuprofen on blood pressure control in hypertensive patients on angiotensin converting enzyme inhibitors. Am J Hypertens, 2003. 16(2): p. 135-9.

51. Paoloni, J.A., et al., Non-steroidal anti-inflammatory drugs in sports medicine: guidelines for practical but sensible use. Br J Sports Med, 2009. 43(11): p. 863-5.

52. Dupuy, O., et al., An Evidence-Based Approach for Choosing Post-exercise Recovery Techniques to Reduce Markers of Muscle Damage, Soreness, Fatigue, and Inflammation: A Systematic Review With Meta-Analysis. Front Physiol, 2018. 9: p. 403.

53. Boonstra, A.M., et al., Reliability and validity of the visual analogue scale for disability in patients with chronic musculoskeletal pain. Int J Rehabil Res, 2008. 31(2): p. 165-9.

54. Swift, A., Physiology-how the body detects pain stimuli. Nurs Times, 2015. 111(39): p. 20-3.

\section{Figures}
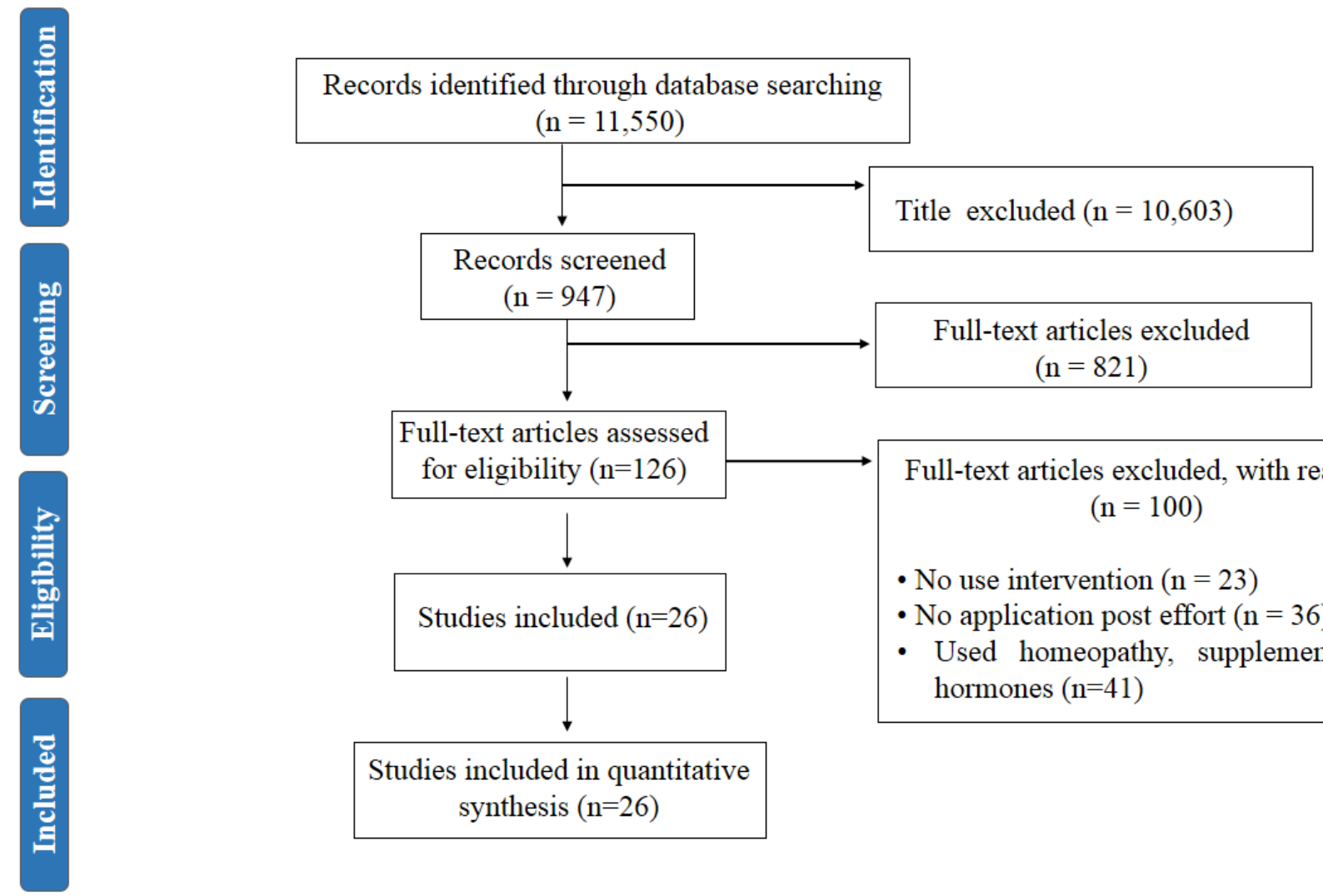

Figure 1

Description of excluded studies according to the established criteria. 


\begin{tabular}{|c|c|c|c|c|c|c|c|}
\hline & 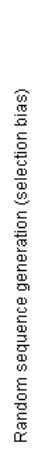 & 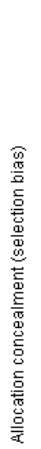 & 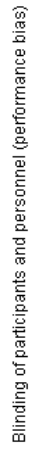 & 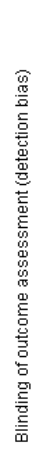 & 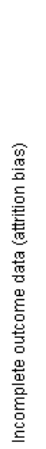 & 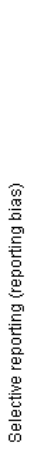 & 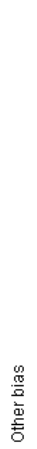 \\
\hline Arendt et al., 2007 & $\odot$ & & $\odot$ & - & & - & \\
\hline Cannavino et al., 2003 & + & $\odot$ & $\odot$ & $\theta$ & & ○ & \\
\hline Croisier et al., 1996 & 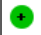 & ○ & † & - & & & ๑ \\
\hline Donnelly et al., 1988 & $\theta$ & ○ & ๑ & - & & & 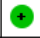 \\
\hline Donnely et al., 1990 & $\odot$ & ○ & ๑ & 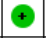 & & & \\
\hline Dudley et al., 1997 & ○ & ○ & & ๑ & 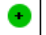 & & \\
\hline Grossman et al., 1995 & $\odot$ & - & $\odot$ & - & & & † \\
\hline Hyldahl et al., 2010 & 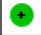 & ○ & & - & & & \\
\hline Krent, et al., 2008 & $\odot$ & - & $\odot$ & $\theta$ & & & \\
\hline Lecomte et al., 1998 & 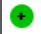 & - & 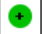 & - & 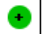 & & ๑ \\
\hline Loram et al., 2005 & 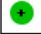 & ○ & $\odot$ & - & $\odot$ & & 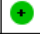 \\
\hline McAnulty et al., 2007 & ○ & ○ & ○ & - & & & అ \\
\hline Nieman et al., 2006 & $\theta$ & - & $\theta$ & - & & & $\theta$ \\
\hline Rahnama et al., 2005 & $\odot$ & ○ & ○ & - & & & \\
\hline Rother et al., 2014 & 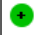 & $\odot$ & & $\odot$ & $\odot$ & & 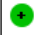 \\
\hline Seidel et al., 2016 & 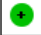 & ○ & $\odot$ & - & $\odot$ & & 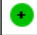 \\
\hline Smith et al., [1] 1995 & ๑ & - & ๑ & - & & & \\
\hline Smith et al., [2] 1995 & ๑ & ○ & 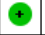 & - & & & \\
\hline Stone et al., 2002 & ๑) & ○ & $\oplus$ & - & & & 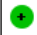 \\
\hline Tokmakidis et al., 2003 & $\odot$ & - & $\odot$ & - & $\odot$ & & \\
\hline Vella et al., 2016 & $\odot$ & - & $\odot$ & - & $\odot$ & & $\odot$ \\
\hline
\end{tabular}

\section{Figure 2}

Bias risk evaluation of the selected studies examining the efficacy of NSAIDs for muscle soreness. Low risk (+), unclear risk (blank) and high risk (-) for different features of the Cochrane Risk of Bias Tool. 


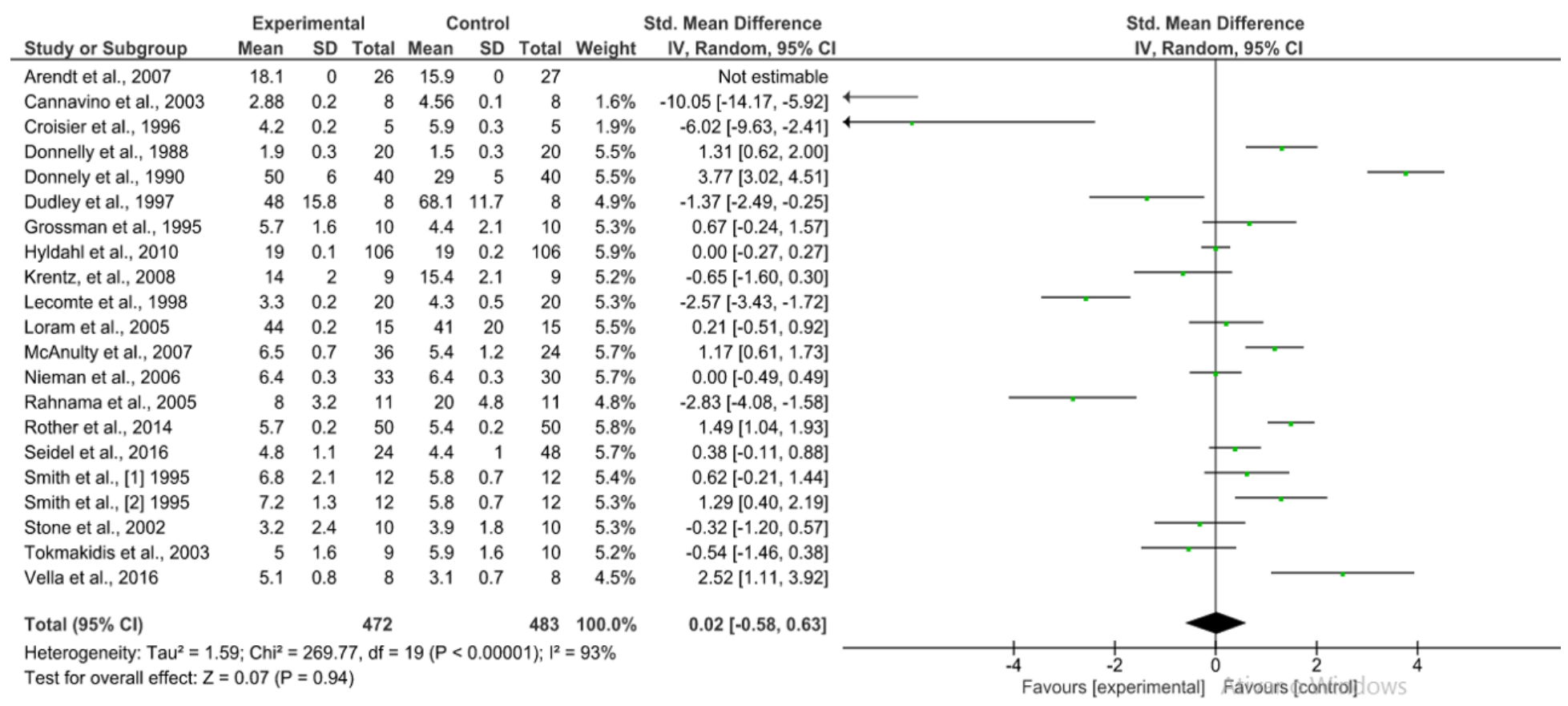

\section{Figure 3}

Forest plot showing the effects of NSAIDs (experimental) versus control condition on the management of DOMS. SD: standard deviation; Std: standardized; Cl: confidence interval. Program: (RevMan, 5.3.5); heterogeneity: Tau2 = 1.59; Ch2 = 2269,77, df = $19(P 0.000001) ; 12=93 \%$. 